\title{
The Relationship Between Rejection Sensitivity and Dormitory Interpersonal Relations in Female College Students: The Mediating Effect of Coping Style
}

\author{
Yongmei $\mathrm{Hou}^{1, *}$ and Tingyan $\mathrm{Li}^{1}$
}

\author{
${ }^{1}$ Department of Psychology, School of Humanity and Administration, Guangdong Medical University, Dongguan \\ 523808, Guangdong Province, China \\ *Corresponding author Email: wdtgxx_1187333 @163.com
}

\begin{abstract}
S
Dormitory interpersonal relationship plays an important role in female undergraduates' learning efficiency, personality growth and life quality. The aim of this study is to explore the status of female undergraduates' rejection sensitivity, coping style and dormitory interpersonal relationship, and analyze the relationships among these 3 variables. 780 female undergraduates were selected with stratified random sampling from 7 colleges in Guangdong Province. They were investigated with Refusal Sensitivity Scale (RSS), Simplified Coping Strategy Questionnaire (SCSQ) and Dormitory Interpersonal Relationships Questionnaire for Female College Students (DIRQFCS). The survey results indicate the following three points. First, the scores of RSS, SCSQ, positive coping (PC), negative coping (NC), DIRQFCS and its four aspects (interpersonal and group knowledge (IGK), interpersonal communication (IC), individual traits (IT) and interpersonal disturbance (ID) were (56.5 \pm 8.7$),(13.6 \pm 6.6),(22.9 \pm 5.7),(9.4 \pm 3.9),(85.4 \pm 8.8),(12.5 \pm 2.2),(16.6 \pm 2.2),(25.0 \pm 3.2)$ and (31.4 \pm 3.2$)$, respectively. Second, apart from PC vs. rejection sensitivity, PC vs. IC, the total score of SCSQ vs. the other 8 variables, there was a significantly pairwise correlation among the other variables. Third, the score of NC played a partial mediating effect in the relationship between the total scores of RSS and DIRQFCS, with the mediating effect accounting for $18.0 \%$ of the total effect. It is therefore suggested that rejection sensitivity not only directly influences the dormitory interpersonal relationship in the female undergraduates, but also indirectly influences it through negative coping.

Keywords: Female College Students, Rejection Sensitivity, Coping Style, Dormitory Interpersonal
\end{abstract}

Relationship, Mediating Effect

\section{INTRODUCTIONS}

The status of dormitory interpersonal relationships among female college students is as much censured as praised. Most studies believe that there are obvious problems: MENG Fanqi [1] and MA Hui [2] find that nearly $50 \%$ of the "post-90s" female college students are not satisfied with the dormitory relationship, and the investigation by LV Yinfang [3] shows that $32.5 \%$ of female college students are "very dissatisfied", "dissatisfied" and "somewhat dissatisfied" with the dormitory relationship while $53.5 \%$ are "somewhat satisfied", "satisfied" and "very satisfied", and 14\% are "indifferent". A few scholars hold an optimistic attitude. SHEN Xiongyong [4] thinks that in spite of irregular interpersonal conflicts in female college students' dormitories, the overall atmosphere is good with the harmony and compatibility higher than the conflict; LI Yanna [5] finds that $87.1 \%$ of female college students are satisfied with dormitory interpersonal relationship. The factors influencing female college students' dormitory interpersonal relationship, which can be summarized into the following categories: (1) demographic factors, such as the source of students [6], dormitory type [7-8], whether is the only child [7], whether is a student cadre [4,7], major [4], grade [3-4], whether has dormitory experience before [3]. (2) personal factors, such as living habits [3], regular schedule [3], interests and hobbies [3], personality [3], bad cognition and emotion [9], conflict of interest and competition [10-11], etc. (3) university management factors, such as lack of scientific and strict student management system, insufficient number of management personnel and weak management abilities $[5,10]$. To sum up, previous studies mostly involve two or three influencing factors of female college students' dormitory interpersonal relationship (especially demographic factors); personal factors are mostly discussed from the aspects of living habits, aggressive personality, jealousy and dissatisfaction, resource competition and so on, focusing on the role of aggression. The theoretical assumption is that female college students are highly confident and gregarious, without noticing such a reality that: some female college students also lack self-confidence and are faced with social barriers. They 
will have interpersonal problems in dormitories because of cognitive bias, one of which is rejection sensitivity.

The definition of rejection sensitivity has not been finalized. Mehrabian (1970) believes that rejection sensitivity is a kind of personality, which is the individual's fear and concern of others' refusal behavior when such situation occurs or is expected to happen, and makes the individual have negative experience, evaluation and response of social relationship. Downed and Feldman et al. propose such idea after reviewing a large number of studies: rejection sensitivity is "individual's expectation of anxiety arising from refusal and tendency to readiness awareness and overreaction" (Downey \& Feldman, 1996). It can be seen that rejection sensitivity is a kind of personality dynamic tendency, which can lead to negative cognitions, emotions and behaviors of individuals, and trigger or strengthen the physical and mental stress response [12]. Leary et al. [12] finds that the rejected individual will have a hostile reaction, which is more intense in individuals with high rejection sensitivity; the research of Ayduk and Downey (1996) shows that when individuals with high rejection sensitivity feel rejected in intimate relationship, they are more likely to fall into interpersonal conflict, and women with high rejection sensitivity are more likely to have hostility to their lovers than men. LI Xia [13] finds that rejection sensitivity is positively correlated with self-esteem, social anxiety and loneliness. SUN Xiaoling [14] finds that rejection sensitivity is positively correlated with social anxiety and negatively correlated with interpersonal trust. ZHENG Mingwu [15] finds that female students' rejection sensitivity has a negative predictive effect on problem solving while males' rejection sensitivity has a positive predictive effect on depression, and rejection sensitivity has a positive predictive effect on complaint behaviors of both male and female students.

Coping style is an effort process of cognition and behavior made by individuals in the face of frustration and stress in order to reduce their impact. It is the intermediary mechanism between stress and mental health, with buffer effect [16].

To sum up, there is such a logical connection between rejection sensitivity, coping style and dormitory interpersonal relationship: rejection sensitivity is a kind of cognitive bias, which can lead to negative cognitions, emotions and behaviors of the individual, which, reflected in the (dormitory) interpersonal interaction, is prone to trigger hostility from such individual because he/she worries (or expects) roommate's rejection, worsening the dormitory interpersonal relationship, and triggering or strengthening physical and mental stress response [12]. Different coping styles can buffer or strengthen such stress response.

Empirical research shows that there are significant pairwise correlations among rejection sensitivity, coping style and dormitory interpersonal relationship [15, 17-20]. It can be assumed that there is a mediating effect among rejection sensitivity, coping style and dormitory interpersonal relationship. Specifically speaking, dormitory interpersonal relationship is an individual's response in behavior and emotion, which is an outcome variable. Rejection sensitivity is a kind of personality, belonging to the deep and remote psychological quality (antecedent variable). The coping style is the individual's way of dealing with life events, which is the surface and proximal psychological quality (antecedent variable). Rejection sensitivity is more mediated by coping style.

Based on the above analysis, it can be assumed that coping style plays a mediating role between rejection sensitivity and dormitory interpersonal relationship.

\section{RESEARCH OBJECT AND TOOLS}

\subsection{Research Object}

\subsubsection{Sample Size Estimation}

The minimum sample size is calculated by $G *$ Power 3 [21]. As the detection rate of dormitory interpersonal relationship problems among domestic female college students is $12.9-49.8 \%$ [1-5], the test effect value is at medium level [1-5], that is, $d$ value is $0.50-0.80$ [22]. In this study, we set the effect value $d=0.70$, the statistical test force $1-\beta=0.80$, the type I error probability $\alpha=0.05$, and the minimum sample size is calculated as 748 . The minimum sample size is determined as 898 due to a $20 \%$ of possible follow-up loss rate.

\subsubsection{Sampling}

Through stratified random sampling, 850 female college students from 7 universities, including Guangdong Medical University, Dongguan University of Technology, Zhongkai University of Agriculture and Engineering, Guangdong University of Finance \& Economics, Guangdong Polytechnic Normal University, Guangzhou Sport University and Xinghai Conservatory of Music, were selected as the research objects. 780 valid questionnaires were collected with the rate of $91.76 \%$. The average age of the objects was $(20.38 \pm 1.50)$ years old; There were 212 freshmen, 211 sophomores, 197 juniors and 160 seniors; 671 only children and 109 non-only children; 322 students from urban or town areas and 458 students from rural areas; 221 living in mixed dormitories and $559(71.67 \%)$ living in non-mixed dormitories; 731 (93.72\%) had dormitory experience before entering university and 49 $(6.28 \%)$ had no dormitory experience; 187 in comprehensive colleges, 153 in science and engineering colleges, 101 in agricultural colleges, 148 in medical colleges, 123 in finance and economics colleges, 24 in sports colleges and 44 in art colleges; 244 majoring in science and engineering, 157 in liberal arts, 171 in pharmaceuticals, 57 in economics, 75 in management, 29 in sports and 47 in arts; 6 from rich, 198 from well-off 
families, 496 from common families, 67 from poor families and 13 from very poor families.

\subsection{Tools}

\subsubsection{Rejection Sensitivity Scale, RSS}

RSS is compiled by Rebecca L Jobe (2003) and revised by LI Xia into Chinese version [13]. For 18 RSS items, the Likert 5-point is used to score from 1 to 5 points corresponding to "completely non-compliant" to "completely compliant". The higher the score, the higher the rejection sensitivity. In this study, the Cronbach's $\alpha$ of the scale is 0.80 .

\subsubsection{Simplified Coping Strategy Questionnaire, $S C S Q$}

Compiled by XIE Yaning (1990) [23], SCSQ has 20 items, divided into two subscales: positive coping (PC) and negative coping (NC). The Likert 4-point is used to score from 0 to 3 points corresponding to 4 choices, namely, "not used", "occasionally used", "sometimes used" and "frequently used". The higher the total score or the score of a certain dimension, the stronger the tendency. In this study, the Cronbach's $\alpha$ of the total scale is 0.89 ; the Cronbach's $\alpha$ of the positive coping and negative coping subscales are 0.85 and 0.81 , respectively.

\subsubsection{Dormitory Interpersonal Relationships Questionnaire for Female College Students, DIRQFCS}

Compiled by NIU Tengque (2014) [24], DIRQFCS has 20 items, divided into four dimensions, namely, interpersonal and group Knowledge (IGK), interpersonal communication (IC), individual traits (IT) and interpersonal disturbance
(ID). The Likert 5-point scoring method is used to score from 1 to 5 points corresponding to "never" to "always". The higher the score, the better the dormitory interpersonal relationship. In this study, the Cronbach's $\alpha$ of the total scale is 0.85 , and the Cronbach's $\alpha$ of dimensions is $0.75-0.81$.

\subsection{Data Processing}

SPSS 20.0 is used to process the valid data. The main statistical methods include descriptive statistics, Pearson correlation analysis and multiple stepwise linear regression analysis.

\section{RESULTS AND ANALYSIS}

\subsection{Overall Status of Rejection Sensitivity, Coping Style and Dormitory Interpersonal Relationship among Female College Students}

The rejection sensitivity of female college students in this group is at the medium level [13], the coping style is positive [23], and the interpersonal relationship in dormitory is good [24].

\subsection{Correlation of Rejection Sensitivity, Coping Style and Dormitory Interpersonal Relationship among Female College Students}

Except for that positive coping v.s rejection sensitivity, positive coping v.s interpersonal communication, total score of SCSQ v.s the other 8 variables are not significantly correlated, the other variables are significantly pairwise correlated (all $\mathrm{P}<.01$ ). The results are shown in Table 1.

Table 1 Descriptive statistics and correlation coefficient of each research variable $(n=780)$

\begin{tabular}{|c|c|c|c|c|c|c|c|c|c|c|}
\hline Variables & $\mathrm{M}(\mathrm{SD})$ & 1 & 2 & 3 & 4 & 5 & 6 & 7 & 8 & 9 \\
\hline 1.RSS total score & $56.5(8.7)$ & & & & & & & & & \\
\hline 2. PC & $29.9(7.5)$ & -.07 & & & & & & & & \\
\hline 3. $\mathrm{NC}$ & $43.0(6.1)$ & $.37 * *$ & $-.09 * *$ & & & & & & & \\
\hline 4. SCSQ total score & $13.6(6.6)$ & $.14 * *$ & $.85^{* *}$ & $-.61 * *$ & & & & & & \\
\hline 5. IGK & $12.5(2.2)$ & $-.27 * *$ & $17 * *$ & $-.17 * *$ & .05 & & & & & \\
\hline 6. IC & $16.6(2.2)$ & $-.20 * *$ & .07 & $.18 * *$ & -.04 & $.50 * *$ & & & & \\
\hline 7. IT & $25.0(3.2)$ & $-38 * *$ & $.12 * *$ & $-.30 * *$ & -.06 & $.47 * *$ & $.54 * *$ & & & \\
\hline 8.ID & $31.4(3.3)$ & $-.30 * *$ & $.09 *$ & $-.26 * *$ & -.07 & $.63 * *$ & $.54 * *$ & $.59 * *$ & & \\
\hline 9.DIRQFC Stotal score & $85.4(8.8)$ & $.37 * *$ & $.13^{* *}$ & $-.29 * *$ & -.05 & $.77 * *$ & $.77 * *$ & $.83 * *$ & $.87 * *$ & \\
\hline
\end{tabular}

Notes: $* * p<0.01$. 


\subsection{The Mediating Effect of Coping Style between Rejection Sensitivity and Dormitory Interpersonal Relationship among Female College Students}

Due to the significant pairwise correlation among RSS total score, NC and DIRQFCS total score, it meets the condition of mediating effect test. Firstly, the scores of RSS, NC and DIRQFCS are decentralized, and then the mediating effects of these three variables are tested according to the method proposed by WEN Zhonglin et al. [23]. The results are shown in Table 2.

Table 2 The Mediating Effect Test of Negative Coping between Rejection Sensitivity and Dormitory Interpersonal Relationship

\begin{tabular}{|c|c|c|c|c|c|c|c|c|c|}
\hline Steps & Dependent variables & Dependent variables & $\beta$ & $S E$ & $B$ & $t$ & $P$ & $R^{2}$ & $R_{\text {adj }}^{2}$ \\
\hline Step 1 (c) & $\begin{array}{l}\text { DIRQFCS total } \\
\text { score }\end{array}$ & RSS total score & -.367 & .034 & -.365 & -10.95 & $<.001$ & .133 & .132 \\
\hline Step2 (a) & $\mathrm{NC}$ & RSS total score. & .162 & 015 & .369 & 11.06 & $<.001$ & 136 & .135 \\
\hline Step3 (c') & DIRQFCS total & RSS total score & -.301 & .036 & -.300 & -5.037 & $<.001$ & .161 & .159 \\
\hline (b) & score & $\mathrm{NC}$ & -.406 & .081 & -.178 & -8.477 & $<.001$ & & \\
\hline
\end{tabular}

According to Table 2, a, b, c and c' are significant. Therefore, the mediating effect of negative coping is partial with the ratio of mediating effect to total effect: effect $\mathrm{m}=\mathrm{ab} / \mathrm{c}=0.369 \times 0.178 / 0.365 \times 100 \%=18.0 \%$.

\section{DISCUSSIONS}

This study shows that the interpersonal relationship of female college students in this research group is generally good, the rejection sensitivity is at the medium level, and the coping style is more positive, which is inconsistent with the results of previous studies [15,17-20]. It may be the result of different sampling. It is suggested that the mental health of female college students has been improved obviously.

There are direct and indirect effects between college students' rejection sensitivity and DIRQFCS total score.

On the one hand, there is a significant negative correlation between female college students' rejection sensitivity and DIRQFCS total score, which is the direct effect between them, consistent with the results of previous studies [17-20]. The higher the score of rejection sensitivity, the worse the dormitory interpersonal relationship. Previous studies have shown that the emotional characteristics, cognitive styles and behavioral patterns of individuals with high rejection sensitivity have negative tendencies [19]. They are more sensitive to rejection. Before having interpersonal interactions, they tend to have excessive worries and negative expectations about others' rejection. After being rejected, they have excessive adverse psychological and behavioral reactions. However, others can't correctly understand this reaction and mistakenly think that their attitude is unfriendly. Therefore, it is easy to cause interpersonal conflicts, hostility and conflicts, which is not conducive to the establishment and maintenance of friendly relations.

On the other hand, there is an indirect effect between female college students' rejection sensitivity and DIRQFCS total score. It is shown as follows: female college students' rejection sensitivity - negative coping DIRQFCS total score, that is, negative coping plays a partial mediating role between female college students' rejection sensitivity and DIRQFCS total score. Those with high rejection sensitivity are more likely to adopt negative coping strategies [14,17-18], such as avoiding roommates and excessive emotional venting, which lead to the accumulation and aggravation of interpersonal problems in dormitories (for example, roommates' anger, hostility, and even aggressive behaviors caused by the avoidance) and further worsen the dormitory interpersonal relationship.

\section{CONCLUSIONS}

This study preliminarily reveals the relationship among female college students' rejection sensitivity, negative coping and dormitory interpersonal relationship, and verifies the following hypothesis: negative coping partially mediates the relationship between female college students' rejection sensitivity and dormitory interpersonal relationship. On the one hand, there is a significant correlation between female college students' rejection sensitivity and dormitory interpersonal relationship, which is the direct effect; on the other hand, there is an indirect effect between female college students' rejection sensitivity and dormitory interpersonal relationship. It is shown as follows: female college students' rejection sensitivity negative coping - dormitory interpersonal relationship, that is, negative coping plays a partial mediating role between female college students' rejection sensitivity and dormitory interpersonal relationship.

To sum up, one of the important reasons for female college students' dormitory interpersonal problems is that the female students with high rejection sensitivity are unable to deal with all kinds of differences, contradictions and conflicts with roommates in a timely and effective manner due to the lack of positive coping styles, which leads to the accumulation and aggravation of problems, thus affecting the dormitory interpersonal relationship. According to the results of this study, we can help female college students form correct self-cognition and interpersonal cognition through attribution training and cognitive correction, reduce their rejection sensitivity, cultivate their positive coping styles, and finally improve their interpersonal 
communication ability and build good dormitory interpersonal relationships.

\section{REFERENCES}

[1] MENG Fanqi, Analysis on the phenomenon of "cold violence" in "post 90s" female college students' dormitories, South Tribune. (4) (2015) pp.86-87. DOI:10.3969/ j.issn. 1004-1133. 2015. 04.030.

[2] MA Hui, Research on female college students' dormitory interpersonal relationship, Heilongjiang Education (higher education research and evaluation). (9)(2010) pp. 28-29.

DOI:10.3969/j.issn.1002-4107.2010.09.013.

[3] LV Yinfang, YAO Jingru, CUI Weidong, Investigation and analysis of female college students' dormitory interpersonal relationship, New Western. (17) (2019) pp. 64-66.

DOI:CNKI:SUN:XXBL.0.2019-17-033.

[4] SHEN Xiongyong, Investigation on dormitory interpersonal harmony of female college students, Journal of Henan Institute of Science and Technology (Social Science Edition). (1) (2014) pp. 107-109. DOI: 10.3969/j.issn.1673-6060.2014.01.028.

[5] LI Yanna, Investigation and research on dormitory interpersonal relationship of female college students, Science and Education Guide. (1) (2013) pp.249-250. DOI: 10.3969/j.issn. 1674-6813.2013.01.141

[6] SHI Xiaoming, Analysis of dormitory interpersonal conflicts among post-90s female college students caused by regional reasons, Journal of Yangzhou College of Education. (3)(2015) pp. 61-64. DOI: 10.15977/j.cnki.cn32-1555/g4.2015.03.015

[7] JIANG Ruichen, Research on dormitory interpersonal relationship among contemporary female college students, Journal of Anqing Normal University (SOCIAL SCIENCE EDITION). (1) (2016)

pp.149-151. DOI:

10.13757/j.cnki.cn34-1045/c.2016.01.035

[8] LUO Qiaohui, Investigation and research on female college students' dormitory interpersonal relationship -Taking Tianjin Normal University as an example, Theory Research. (20) (2011) pp.41-42. DOI: 10.3969/j.issn.1002-2589.2011.20.021

[9] ZHANG Fan, Research and analysis of dormitory interpersonal relationship among female college students, Data of Culture and Education. (18) (2017) pp.150-152. DOI:10.3969/j.issn.1004-8359.2017.18.070
[10] SHI Yujing, Research on dormitory interpersonal relationship among "post-90s" female college students, Talent Resource Development. (16) (2015) p. 202. DOI: 10.3969/j.issn. 1003 - 1073.2015.16.152

[11] SHEN Zhaoxia, SHI Xiaoming, Analysis and mediation of dormitory interpersonal conflicts among "post-90s" female college students, Journal of Changchun Institute of Education. 32 (1)(2016) pp.13-15. DOI: 10.3969/j.issn.1671-6531.2016.01.004

[12] ZHANG Yingrui, XIAO Yingxia, Psychological mechanism of rejection sensitivity and its related researches, China Journal of Health Psychology. 26(8)(2018) pp.1273-1277. DOI: 10. 13342/j.cnki.cjhp.2018.08.040

[13] LI Xia, Correlation research of rejection sensitivity, Ma.D. Thesis, Jiangxi Normal University, 2007. DOI: $10.7666 /$ d.y1224003

[14] SUN Xiaoling, WU Mingzheng, Relationships between self-esteem, rejection sensitivity, interpersonal trust and social anxiety in college students, Chinese Journal of Clinical Psychology. 19(4)(2011)pp. 537-539. DOI: CNKI:SUN:ZLCY.0.2011-04-034

[15] ZHENG Mingwu, Relationships between rejection sensitivity, emotional intelligence and coping style of college students, Ma.D. Thesis, Jiangxi Normal University, 2012. DOI: 10.7666/d.D367832

[16] Compas, Bruce E, Coping with Stress during Children and Adolescence: Problems, Progress, and Potential in Theory and Research, Psychological Bulletin. 3(101)(1987) pp.393-403. DOI: 10.1037/0033-2909.101.3.393

[17] LIAO Jun, ZHOU Bo, LUO Cheng, YU Qiumei, A study on the relationship between freshmen's coping style and dormitory interpersonal distress, Chinese Journal of Health Psychology. 20(1)(2012) pp.114-116. DOI:10.13342/j.cnki.cjhp.2012.01.033

[18] LIAO Jun, The influence of freshmen's coping style on dormitory interpersonal relationship and the moderating effect of gender, Campus Psychology. 16(6)(2018) pp.35-38.

DOI:10.19521/j.cnki.1673-1662.2018.06.011

[19] CHEN Jiawei, Research on interpersonal relationship of post-90s college students and its influence on rejection sensitivity and social anxiety, Journal of Minnan Normal University (NATURAL SCIENCE EDITION). (4)(2012) pp.123-126. DOI: CNKI:CDMD:2.1013.349431 
[20] LI Jie, A study on the relationship among self-esteem, rejection sensitivity and interpersonal relationship of postgraduates, Ma.D. Thesis, Fujian Normal University, 2013. DOI: CNKI:

CDMD:2.1013.349431

[21] Cohen Jacob, Statistical power analysis, Curr Direct Psychol Sci. 1(13)(1992)pp. 98-101.

DOI:10.1111/1467-8721.ep10768783

[22] ZHANG Houcan, XU Jianping, Modern Psychology and Education Statistics, Beijing Normal University Publishing Group, 2012.
[23] WANG Xiangdong, WANG Xilin, MA Hong, Handbook of Mental Health Assessment Scale (Revised edition), Chinese Mental Health Journal, 1999.

[24] NIU Tengque, Compilation and application of interpersonal relationship questionnaire for female college students, Ma.D. Thesis, Shanxi Normal University, 2014. DOI: CNKI: CDMD: 2.1014.225885

[25] WEN Zhonglin, HOU Jietai, ZHANG Lei, Comparison and application of regulatory effect and mediating effect, Acta psychologica Sinica. 37(2)(2005) pp.268-274. DOI: CNKI: SUN: XLXB.0.2005-02-00F 